\title{
A CLASS OF ESTIMATORS OF THE SURVIVAL FUNCTION FROM INTERVAL-CENSORED DATA ${ }^{1}$
}

\author{
By Zhiming Wang and Joseph C. Gardiner \\ Michigan State University
}

\begin{abstract}
A model of interval censorship of a failure time $T$ is considered when there is only one inspection time $Y$. The observable data are $n$ independent copies of the pair $(Y, \delta)$, where $\delta=[T \leq Y]$. We construct a class of self-consistent estimators of the survival function of $T$ defined implicitly through two equations and show their strong consistency under certain conditions. The properties of the nonparametric maximum likelihood estimator are also investigated.
\end{abstract}

1. Introduction. Some epidemiologic investigations and natural history studies of infectious diseases are characterized by periodic examination of subjects for events of interest. The exact occurrence time $T$ of an event is not observed, but what is known is the interval in which the event took place. Therefore $T$ is said to be interval censored, with the censoring interval obtained from the pattern of examination times. It is possible that the event in question may not have occurred by the time of the last examination in which case $T$ is right censored. Several examples of studies where intervalcensored data arise naturally have been reported, particularly in connection with the time of seroconversion or manifestation of AIDS in subjects exposed to the human immunodeficiency virus. Since only periodic assessment of patients is feasible, the time of seroconversion will be recorded to within an interval specified by the last negative and the first positive assessment. Where no positive assessment was made by the time of the last examination, the time of seroconversion will be right censored. In assessing the incidence of intraventricular hemorrhage, a brain lesion that is common in preterm infants, Pinto-Martin, et al. (1992) report on a study that ascertained hemorrhage status by cranial ultrasonography at three times in the first week of life in a large cohort of low-birthweight infants. The time of onset of hemorrhage $T$ is interval censored because it can only be specified to within an interval between the first positive and last negative ultrasound scan. Estimation of the distribution of $T$ from these data utilizing the nonparametric scheme of Turnbull (1976) is reported in Paneth, et al. (1993). For other examples from medicine, see Rücker and Messerer (1988) and Peckham (1991).

Received April 1994; revised July 1995.

${ }^{1}$ Research supported in part by the NIH under Grant 1-R01-GM-49374.

AMS 1991 subject classifications. Primary 60G05; secondary 62G99.

Key words and phrases. Interval censorship, self-consistency, EM algorithm, counting process. 
Estimation of the distribution of $T$ from interval-censored observations has become the focus of intensive research recently. Groeneboom and Wellner (1992) obtain the nonparametric maximum likelihood estimator (NPMLE) in two models of interval censorship. The first model, called Case I, considers a single inspection time $Y$ and the datum on each subject is $(Y, \delta)$, where $\delta=[T \leq Y]$ is the indicator of the displayed event $T \leq Y$. In this case $T$ is either left censored $(\delta=1)$ or right censored $(\delta=0)$. The second model, called Case II, allows for two examination times. Wang, Gardiner and Ramamoorthi (1994) have shown the identifiability of the distribution of $T$ in two models of interval censorship, one that considers a fixed number of examinations per subject and another in which the follow-up period is fixed but the number of inspections made on a subject is random. Rabinowitz, Tsiatis and Aragon (1995) have addressed estimation in regression models with interval-censored observations arising from multiple inspections on the subjects. Turnbull (1976) first developed an algorithm for computing an estimator of the survival function from interval-censored observations. His method of estimation is based on maximum likelihood considerations and yields a system of equations (self-consistent equations) that may be solved using the EM algorithm. Groeneboom and Wellner (1992) provide a remarkably elegant development of the asymptotic properties of the NPMLE in Cases I and II that exploits the connection between NPMLE and the least squares isotonic regression estimate.

This article focuses on a class of estimators of the distribution of $T$ in the single inspection model of interval censorship. An example of this single inspection model comes from bioassay or toxicity studies where $Y$ denotes the dose of a drug assigned to a subject and $T$ its tolerance level. In some carcinogenicity studies $T$ denotes the time of tumor appearance in animals that have been exposed to a carcinogen and $Y$ the time of death or sacrifice of the animal.

This article introduces a class of estimators defined implicitly through two equations, which we demonstrate are equivalent to the solution of the selfconsistency equation (Theorem 3.1). Although the solution to our equation is not unique, the NPMLE is shown to satisfy it. Under a certain condition that is also met by the NPMLE, we establish the strong consistency of our estimators (Theorem 4.1). However, Groeneboom and Wellner (1992) have a direct proof of the consistency of the NPMLE.

The substantive material of this paper is divided as follows. Section 2 introduces the basic notation used in the sequel and the definition of our sequence of estimators. Section 3 is devoted to proving the self-consistency of these estimates followed by Section 4 which addresses their strong consistency.

2. Notation and definition of estimators. Let the nonnegative random variable $T$ be an event time with survival function $S(t)=P(T>t)$ and $Y$ be the inspection time on $R^{+}=[0, \infty)$ with survival function $S_{Y}(t)=$ $P(Y>t)$. Observation is confined to the pair $(Y, \delta)$, with $\delta=[T \leq Y]$, 
in $n$ independent and identically distributed copies of $(Y, \delta)$, denoted $\left\{\left(Y_{i}, \delta_{i}\right): i=1,2, \ldots, n\right\}$. The following conditions are assumed to hold in the sequel:

(A1) $T$ and $Y$ are independent.

(A2) $S_{Y}$ is continuous, and $S_{Y}(s)-S_{Y}(t)>0$ for all $0 \leq s<t<\infty$.

(A3) $S$ is continuous, and $S(s)-S(t)>0$ for all $0 \leq s<t<\infty$.

We define the subdistributions, $W_{i}, i=1,2$, by

$$
W_{1}(t)=P(Y>t, \delta=1) \quad \text { and } \quad W_{2}(t)=P(Y>t, \delta=0), \quad t \in[0, \infty),
$$

which can be written in terms of the survival functions as

$$
\begin{aligned}
& W_{1}(t)=-\int_{t}^{\infty}(1-S(s)) d S_{Y}(s), \\
& W_{2}(t)=-\int_{t}^{\infty} S(s) d S_{Y}(s) .
\end{aligned}
$$

The two counting processes $N_{L}$ and $N_{R}$ corresponding to left and right censorship are given by

$$
N_{L}(t)=\sum_{i=1}^{n}\left[Y_{i} \leq t, \delta_{i}=1\right] \quad \text { and } \quad N_{R}(t)=\sum_{i=1}^{n}\left[Y_{i} \leq t, \delta_{i}=0\right] .
$$

Then $W_{1}^{(n)}(t)=(1 / n)\left(N_{L}(\infty)-N_{L}(t)\right)$ and $W_{2}^{(n)}(t)=(1 / n)\left(N_{R}(\infty)-N_{R}(t)\right)$ are the empirical processes corresponding to (2.1) and (2.2). The empirical survival distribution of the examination times $Y_{1}, \ldots, Y_{n}$ is denoted by

$$
\tilde{S}_{Y}^{(n)}(t)=\frac{1}{n} \sum_{i=1}^{n}\left[Y_{i}>t\right]
$$

Throughout we adhere to the following convention on denoting integrals that would allow us to write many expressions in a less cumbersome form: $\int_{(t, \infty)}$ is $\int_{t+}^{\infty}, \int_{[t, \infty)}$ is $\int_{t}^{\infty}$ and $\int_{[0, t]}$ is $\int_{0}^{t}$. Let $\lambda$ be Lebesgue measure on $[0, \infty)$ and by convention we take $0 / 0=0$. A function on $[0, \infty)$ is said to be a subsurvival function if it is nonnegative, nonincreasing and right continuous. It is called a survival function if, additionally, its values at 0 and $\infty$ are 1 and 0 , respectively.

DEFINITION OF ESTIMATORS. We construct two sequences $S^{(n)}$ and $S_{Y}^{(n)}$ implicitly through

$$
\begin{aligned}
& W_{1}^{(n)}(t)+\int_{t+}^{\infty} \frac{1-S^{(n)}(t)}{1-S^{(n)}(s)} d W_{1}^{(n)}(s)=-\int_{t+}^{\infty} S_{Y}^{(n)}(s-) d S^{(n)}(s), \\
& W_{2}^{(n)}(t)=-\int_{t+}^{\infty} S^{(n)}(s) d S_{Y}^{(n)}(s),
\end{aligned}
$$

with $S^{(n)}(0)=S_{Y}^{(n)}(0)=1, t \in[0, \infty)$. 
In the next section, we will show that a solution $S^{(n)}$ exists and that it is a self-consistent estimator of $S$, but first some important remarks regarding (2.3) and (2.4) are in order.

REMARKs. (a) An obvious approach to obtaining estimators of $S$ and $S_{Y}$ is to replace all functions in (2.1) and (2.2) by their estimators, and search for a solution to the equations. For this approach, we would solve for $S^{(n)}$ and $S_{Y}^{(n)}$ from the equations:

$$
\begin{aligned}
& W_{1}^{(n)}(t)=-\int_{t+}^{\infty}\left(1-S^{(n)}(s)\right) d S_{Y}^{(n)}(s), \\
& W_{2}^{(n)}(t)=-\int_{t+}^{\infty} S^{(n)}(s) d S_{Y}^{(n)}(s) .
\end{aligned}
$$

This scheme can be applied to both right censorship and double censorship using expressions analogous to (2.1) and (2.2) in those cases. The method was used by Chang and Yang (1987) to obtain consistent estimates of $S$ in the case of double censorship. However, in our case of interval censoring, if we solve for $S^{(n)}$ and $S_{Y}^{(n)}$ from the above equations, then $S_{Y}^{(n)}$ is easily seen to be the empirical survival distribution $\tilde{S}_{Y}^{(n)}$ of $S_{Y}$ but $S^{(n)}$ will not be a survival function. The modification made in (2.3) leads to an appropriate solution of $S^{(n)}$ as a survival function.

(b) Although $S_{Y}^{(n)}$ in (2.3) and (2.4) is not the empirical $\tilde{S}_{Y}^{(n)}$, it is very closely related to it. Later, in Section 4 , we obtain a relationship between $S_{Y}^{(n)}$ and $\tilde{S}_{Y}^{(n)}$. See also (3.4) for an explicit definition of $S_{Y}^{(n)}$ in terms of $S^{(n)}$.

(c) The estimators $S^{(n)}$ and $S_{Y}^{(n)}$ are specified only at the observed points $\left\{Y_{i}: 1 \leq i \leq n\right\}$. We may extend them in a natural way to be right-continuous step functions with jumps only at the $Y_{i}$ 's.

3. Self-consistent estimators. Given the information ( $\sigma$-field), $\sigma\left\{\left(Y_{i}, \delta_{i}\right): i=1,2, \ldots, n\right\}=\mathscr{A}_{n}$, computation of the conditional expectation $E\left((1 / n) \sum_{i=1}^{n}\left[T_{i} \leq t\right] \mid \mathscr{A}_{n}\right)$ gives

$$
\iint\left\{\frac{F(t \wedge u)}{F(u)}[x \leq u]+\frac{F(t)-F(t \wedge u)}{1-F(u)}[x>u]\right\} d P_{n}(x, u),
$$

where

$$
P_{n}(x, u)=\frac{1}{n} \sum_{i=1}^{n}\left[T_{i} \leq x, Y_{i} \leq u\right]
$$

and $F=1-S$. A self-consistent estimator of $S$ is a sequence $S^{(n)}$ that satisfies the equation (self-consistency equation):

$$
S^{(n)}(t)=E\left(\frac{1}{n} \sum_{i=1}^{n}\left[T_{i}>t\right] \mid \mathscr{A}_{n}\right),
$$

where the right-hand side is evaluated at $S^{(n)}$. The next theorem establishes the equivalence between the self-consistent estimator and the solution to (2.3) and (2.4). 
THEOREM 3.1. If $S^{(n)}$ is a self-consistent estimator of $S$, then there exists a survival function $S_{Y}^{(n)}$, such that $S^{(n)}$ and $S_{Y}^{(n)}$ satisfy (2.3) and (2.4). Conversely, if $S^{(n)}$ and $S_{Y}^{(n)}$ are a solution of (2.3) and (2.4), then $S^{(n)}$ is a self-consistent estimator of $S$.

Proof. Suppose $S^{(n)}$ is a self-consistent estimator of $S$. Then

$$
1-S^{(n)}(t)=E_{S^{(n)}}\left\{F_{n}(t) \mid Y_{1}, \ldots, Y_{n} ; \delta_{1}, \ldots, \delta_{n}\right\},
$$

where $F_{n}(t)=(1 / n) \sum_{i=1}^{n}\left[T_{i} \leq t\right]$ is the empirical distribution of $F=1-S$, and $E_{S^{(n)}}$ is the expectation under the assumption that the $T_{i}$ have the survival distribution $S^{(n)}$ for any $i$. Hence

$$
\begin{aligned}
1-S^{(n)}(t)= & \frac{1}{n} \sum_{i=1}^{n} E_{S^{(n)}}\left\{\left[T_{i} \leq t\right] \mid Y_{i}, \delta_{i}\right\} \\
= & \frac{1}{n} \sum_{i=1}^{n}\left\{\frac{1-S^{(n)}\left(t \wedge Y_{i}\right)}{1-S^{(n)}\left(Y_{i}\right)} \delta_{i}+\frac{S^{(n)}\left(t \wedge Y_{i}\right)-S^{(n)}(t)}{S^{(n)}\left(Y_{i}\right)}\left(1-\delta_{i}\right)\right\} \\
= & \frac{1}{n}\left[N_{L}(t)+\int_{t+2)}^{\infty} \frac{1-S^{(n)}(t)}{1-S^{(n)}(s)} d N_{L}(s)\right. \\
& \left.\quad+\int_{0}^{t} \frac{S^{(n)}(s)-S^{(n)}(t)}{S^{(n)}(s)} d N_{R}(s)\right] .
\end{aligned}
$$

Using the definition of $W_{1}^{(n)}$ and $W_{2}^{(n)}$ and the fact that $N_{L}(\infty)+N_{R}(\infty)=n$, we get

$$
\begin{aligned}
S^{(n)}(t)= & W_{1}^{(n)}(t)+W_{2}^{(n)}(t)+\int_{t+}^{\infty} \frac{1-S^{(n)}(t)}{1-S^{(n)}(s)} d W_{1}^{(n)}(s) \\
& -\int_{0}^{t} \frac{S^{(n)}(t)}{S^{(n)}(s)} d W_{2}^{(n)}(s) .
\end{aligned}
$$

Now define an estimator of $S_{Y}$ by

$$
S_{Y}^{(n)}(t)=1+\int_{0}^{t} \frac{1}{S^{(n)}(s)} d W_{2}^{(n)}(s), \quad t \in[0, \infty) .
$$

The definition of $S_{Y}^{(n)}$ is valid, since $S^{(n)}(t) \geq W_{2}^{(n)}(t)$ by (3.3) and $0 / 0=0$. Also, $S_{Y}^{(n)}$ is a right-continuous nonincreasing function on $[0, \infty)$ with $S_{Y}^{(n)}(0)=$ 1. Differentiating (3.4) yields

$$
d S_{Y}^{(n)}(s)=\frac{1}{S^{(n)}(s)} d W_{2}^{(n)}(s) \quad \text { or } \quad d W_{2}^{(n)}(s)=S^{(n)}(s) d S_{Y}^{(n)}(s)
$$

Integrating (3.5) leads to (2.4).

Let $Y_{(n)}$ be the largest of the inspection times $Y_{1}, \ldots, Y_{n}$ and $Y_{(n+1)}>Y_{(n)}$ be an arbitrary point on which the remaining mass of $S^{(n)}$ and $S_{Y}^{(n)}$ is placed. 
Then $S^{(n)}(\infty)=S_{Y}^{(n)}(\infty)=0$. This does not affect the self-consistency of $S^{(n)}$. Then (3.3) becomes

$$
\begin{aligned}
S^{(n)}(t)= & W_{1}^{(n)}(t)-\int_{t+}^{\infty} S^{(n)}(s) d S_{Y}^{(n)}(s)+\int_{t+}^{\infty} \frac{1-S^{(n)}(t)}{1-S^{(n)}(s)} d W_{1}^{(n)}(s) \\
& -S^{(n)}(t) \int_{0}^{t} d S_{Y}^{(n)}(s)
\end{aligned}
$$

with $S_{Y}^{(n)}(0)=1$. Using the integration-by-parts formula $d(U V)=V d U+$ $U_{-} d V$ for discontinuous functions $U$ and $V$, this becomes

$$
W_{1}^{(n)}(t)+\int_{t+}^{\infty} \frac{1-S^{(n)}(t)}{1-S^{(n)}(s)} d W_{1}^{(n)}(s)=-\int_{t+}^{\infty} S_{Y}^{(n)}(s-) d S^{(n)}(s),
$$

which is (2.3). Hence we have shown that the self-consistent estimator $S^{(n)}$ of (3.1) satisfies (2.3) and (2.4).

Conversely, the entire argument above can be reversed. Suppose $S^{(n)}$ and $S_{Y}^{(n)}$ are a solution of (2.3) and (2.4) with $S^{(n)}(0)=S_{Y}^{(n)}(0)=1$ and $S^{(n)}(\infty)=$ $S_{Y}^{(n)}(\infty)=0$. Then we will get (3.4) from (2.4), and substituting it in (2.3) will lead to (3.3), which is the self-consistency equation (3.1).

As noted previously, the estimators $S^{(n)}$ and $S_{Y}^{(n)}$ defined through (2.3) and (2.4) have mass only at the inspection points $\left\{Y_{i}: 1 \leq i \leq n\right\}$, but not necessarily at all points. They may be extended to be right-continuous step functions with jumps at these $Y_{i}^{\prime}$ 's. Unlike the right-censoring and double-censoring cases wherein estimators of $S$ are defined through expressions analogous to (2.3) and (2.4), the self-consistent estimator obtained here from intervalcensored data is not unique. See Chang and Yang (1987) for the doublecensorship case. Both Turnbull (1976) and Groeneboom and Wellner (1992) obtain an NPMLE by maximizing $\prod_{i=1}^{n}\left\{F\left(Y_{i}\right)\right\}^{\delta_{i}}\left\{1-F\left(Y_{i}\right)\right\}^{1-\delta_{i}}$, and they show the NPMLE to be self-consistent and unique under the conventions of right continuity stated here.

The following is an example of a self-consistent estimator which is not the NPMLE.

EXAmPLE 3.1. Suppose we have the following values for $(Y, \delta)$ on $n=5$ subjects: $(1,1),(2,0),(3,1),(4,1)$ and $(5,0)$. The NPMLE may be obtained by direct optimization of the likelihood using the intervals $(0,1],(2,3]$ and $(5, \infty)$ to introduce pseudoparameters $\theta_{1}, \theta_{2}, \theta_{3}$, where $\theta_{1}=P[T \leq 1], \theta_{2}=$ $P[2<T \leq 3], \theta_{3}=P[T>5]$ with $\theta_{1}+\theta_{2}+\theta_{3}=1$. The MLE's of these parameters are $\theta_{1}=1 / 2, \theta_{2}=1 / 6$ and $\theta_{3}=1 / 3$ which yields the right-continuous NPMLE $S_{1}^{(n)}$ below. (The arbitrary point $Y_{(6)}=6$ is used to place the unassigned mass.) It can easily be verified that $S_{1}^{(n)}$ and the estimator $S_{2}^{(n)}$ 
below both satisfy (3.3) which makes them self-consistent estimators. However, $S_{2}^{(n)}$ is not the NPMLE.

$$
S_{1}^{(n)}(t)=\left\{\begin{array}{lll}
1, & t \in[0,1), \\
1 / 2, & t \in[1,3), \\
1 / 3, & t \in[3,6), \\
0, & t \in[6, \infty),
\end{array} \quad S_{2}^{(n)}(t)= \begin{cases}1, & t \in[0,1), \\
2 / 5, & t \in[1,6), \\
0, & t \in[6, \infty) .\end{cases}\right.
$$

4. Strong consistency. We already defined $S, S_{Y}, W_{1}, W_{2}, S^{(n)}, S_{Y}^{(n)}$, $W_{1}^{(n)}, W_{2}^{(n)}$ and $\tilde{S}_{Y}^{(n)}$. They are either survival or subsurvival functions on $[0, \infty)$ and their relationships are given in (2.1), (2.2), (2.3) and (2.4). We will first show that the left limits of $S_{Y}^{(n)}$ and $\tilde{S}_{Y}^{(n)}$ agree at the jump points of $S^{(n)}$.

A self-consistent estimator $S^{(n)}$ of (3.1) is a nonincreasing right-continuous step function. Let $J_{n}=\left\{t_{k}: k=1,2, \ldots, m\right\}$ be the set of jump points of $S^{(n)}$. Let $0=t_{0}<t_{1}<\cdots<t_{m}<\infty$. Then $\left\{t_{k}: k=1,2, \ldots, m\right\} \subset\left\{Y_{i}: i=\right.$ $1,2, \ldots, n\}$. Define

$$
V_{1}^{(n)}(t)=W_{1}^{(n)}(t)+\int_{t+}^{\infty} \frac{1-S^{(n)}(t)}{1-S^{(n)}(s)} d W_{1}^{(n)}(s) .
$$

Then (2.3) becomes

$$
V_{1}^{(n)}(t)=-\int_{t+}^{\infty} S_{Y}^{(n)}(s-) d S^{(n)}(s) .
$$

Since $W_{1}^{(n)}(t)+W_{2}^{(n)}(t)=\tilde{S}_{Y}^{(n)}(t)$, and using (3.5), we get

$$
\begin{aligned}
d W_{1}^{(n)}(t) & =d \tilde{S}_{Y}^{(n)}(t)-d W_{2}^{(n)}(t) \\
& =d \tilde{S}_{Y}^{(n)}(t)-S^{(n)}(t) d S_{Y}^{(n)}(t) \\
& =d\left(\tilde{S}_{Y}^{(n)}(t)-S_{Y}^{(n)}(t)\right)+\left(1-S^{(n)}(t)\right) d S_{Y}^{(n)}(t) .
\end{aligned}
$$

Differentiating both sides of (4.1) and using integration by parts yields

$$
\begin{aligned}
d V_{1}^{(n)}(t)= & d W_{1}^{(n)}(t)-d S^{(n)}(t) \int_{t}^{\infty} \frac{d W_{1}^{(n)}(s)}{1-S^{(n)}(s)}-\frac{1-S^{(n)}(t)}{1-S^{(n)}(t)} d W_{1}^{(n)}(t) \\
= & -d S^{(n)}(t) \int_{t}^{\infty} \frac{d W_{1}^{(n)}(s)}{1-S^{(n)}(s)} \\
= & -d S^{(n)}(t) \int_{t}^{\infty} \frac{d\left(\tilde{S}_{Y}^{(n)}(s)-S_{Y}^{(n)}(s)\right)}{\left(1-S^{(n)}(s)\right)} \\
& +d S^{(n)}(t) \int_{t}^{\infty} \frac{1-S^{(n)}(s)}{1-S^{(n)}(s)} d S_{Y}^{(n)}(s) \\
= & -d S^{(n)}(t) \int_{t}^{\infty} \frac{d\left(\tilde{S}_{Y}^{(n)}(s)-S_{Y}^{(n)}(s)\right)}{\left(1-S^{(n)}(s)\right)}+d S^{(n)}(t) S_{Y}^{(n)}(t-)
\end{aligned}
$$


From (4.2), $d V_{1}^{(n)}(t)=S_{Y}^{(n)}(t-) d S^{(n)}(t)$ and so (4.4) gives

$$
d S^{(n)}(t) \int_{t}^{\infty} \frac{d\left(\tilde{S}_{Y}^{(n)}(s)-S_{Y}^{(n)}(s)\right)}{\left(1-S^{(n)}(s)\right)}=0, \quad t \geq 0 .
$$

Since $d S^{(n)}\left(t_{k}\right) \neq 0$ at the points of jump $t_{k}$ of $S^{(n)},(4.5)$ implies

$$
\int_{t_{k}}^{\infty} \frac{d\left(\tilde{S}_{Y}^{(n)}(s)-S_{Y}^{(n)}(s)\right)}{\left(1-S^{(n)}(s)\right)}=0, \quad k=1,2, \ldots, m .
$$

Hence

$$
\int_{\left[t_{k-1}, t_{k}\right)} \frac{d\left(\tilde{S}_{Y}^{(n)}(s)-S_{Y}^{(n)}(s)\right)}{\left(1-S^{(n)}(s)\right)}=0 \text { for each } k=1,2, \ldots, m,
$$

and since $S^{(n)}$ is constant on $\left[t_{k-1}, t_{k}\right)$,

$$
\frac{1}{1-S^{(n)}\left(t_{k-1}\right)} \int_{\left[t_{k-1}, t_{k}\right)} d\left(\tilde{S}_{Y}^{(n)}(s)-S_{Y}^{(n)}(s)\right)=0,
$$

which implies

$$
\int_{t_{k}}^{\infty} d\left(\tilde{S}_{Y}^{(n)}(s)-S_{Y}^{(n)}(s)\right)=0 .
$$

Therefore,

$$
S_{Y}^{(n)}\left(t_{k}-\right)=\tilde{S}_{Y}^{(n)}\left(t_{k}-\right), \quad k=1,2, \ldots, m,
$$

which shows that the left limits of $S_{Y}^{(n)}$ and $\tilde{S}_{Y}^{(n)}$ agree at every point of jump of $S^{(n)}$.

To obtain the strong consistency of a sequence of self-consistent estimators $S^{(n)}$, we need to assign some condition on the (random) set $J_{n}$ of points of jump of $S^{(n)}$. For any $t>0$ and $\varepsilon>0$, let $A_{t, \varepsilon}=\{\omega: \exists N$ such that, for any $\left.n>N, J_{n}(\omega) \cap(t-\varepsilon, t+\varepsilon) \neq \varnothing\right\}$.

Condition C. For each $t>0$ and $\varepsilon>0, P\left(A_{t, \varepsilon}\right)=1$.

The above condition also means that, for each $\omega$ outside some $P$-null set and $t>0$ and $\varepsilon>0$, there exists $N=N(t, \varepsilon, \omega)$ such that, for any $n>N$, $J_{n}(\omega) \cap(t-\varepsilon, t+\varepsilon) \neq \varnothing$. Therefore, almost surely, in any neighborhood of $t$, we can always find points of jump of $S^{(n)}$ for $n$ large enough. Since we are assuming $S$ to be strictly decreasing, Condition $C$ is also necessary for the almost sure uniform convergence of the sequence of random step functions $S^{(n)}$ to $S$. We will show in Theorem 4.2 that the NPMLE satisfies Condition C. We now establish the strong consistency of a sequence of self-consistent estimators $S^{(n)}$. 
Theorem 4.1. Under Condition $\mathrm{C}, S^{(n)}$ uniformly converges to $S$ almost surely. That is,

$$
P\left(\lim _{n \rightarrow \infty} \sup _{t \in[0, \infty)}\left|S^{(n)}(t)-S(t)\right|=0\right)=1 .
$$

The proof of the theorem will follow from several auxiliary results which are stated as lemmas below. First, we observe that, since $\left\{S^{(n)}(t)\right\}$ and $\left\{S_{Y}^{(n)}(t)\right\}$ are sequences of survival functions, by Helly's theorem, there exists a subsequence $\left\{S^{\left(n^{\prime}\right)}(t), S_{Y}^{\left(n^{\prime}\right)}(t)\right\}$ and subsurvival functions $S^{0}(t)$ and $S_{Y}^{0}(t)$ such that $S^{\left(n^{\prime}\right)} \rightarrow S^{0}$ at continuity points of $S^{0}$ and $S_{Y}^{\left(n^{\prime}\right)} \rightarrow S_{Y}^{0}$ at continuity points of $S_{Y}^{0}(t)$. Also, with probability $1, W_{1}^{(n)}(t) \rightarrow W_{1}(t), W_{2}^{(n)}(t) \rightarrow W_{2}(t)$ and $\tilde{S}_{Y}^{(n)}(t) \rightarrow S_{Y}(t)$ uniformly for $t \in[0, \infty)$, since $W_{1}, W_{2}$ and $S_{Y}$ are continuous. Hence, without loss of generality, we may assume uniform convergence on the whole space $\Omega$. Furthermore, since we will show every subsequence has the same limit, we will assume $\left\{n^{\prime}\right\}=\{n\}$.

LeMma 4.1. $S_{Y}^{0}(t)$ is continuous on $[0, \infty)$.

Proof. From (3.4),

$$
S_{Y}^{(n)}(t)=1+\int_{0}^{t} \frac{1}{S^{(n)}(s)} d W_{2}^{(n)}(s), \quad t \in[0, \infty) .
$$

For continuity points of $S_{Y}^{0}(t) 0 \leq s_{1}<s_{2}$, we have

$$
\begin{aligned}
-\left(S_{Y}^{(n)}\left(s_{2}\right)-S_{Y}^{(n)}\left(s_{1}\right)\right) & =-\int_{\left(s_{1}, s_{2}\right]} \frac{1}{S^{(n)}(s)} d W_{2}^{(n)}(s) \\
& \leq \frac{-\left(W_{2}^{(n)}\left(s_{2}\right)-W_{2}^{(n)}\left(s_{1}\right)\right)}{S^{(n)}\left(s_{2}\right)} \\
& \leq \frac{-\left(W_{2}^{(n)}\left(s_{2}\right)-W_{2}^{(n)}\left(s_{1}\right)\right)}{W_{2}^{(n)}\left(s_{2}\right)}
\end{aligned}
$$

where the last inequality follows from $S^{(n)}\left(s_{2}\right) \geq W_{2}^{(n)}\left(s_{2}\right)$ by (3.3). Letting $n \rightarrow \infty$,

$$
-\left(S_{Y}^{0}\left(s_{2}\right)-S_{Y}^{0}\left(s_{1}\right)\right) \leq \frac{-\left(W_{2}\left(s_{2}\right)-W_{2}\left(s_{1}\right)\right)}{W_{2}\left(s_{2}\right)} .
$$

Hence $S_{Y}^{0}$ is continuous on $[0, \infty)$ by the continuity of $W_{2}$. Therefore, $S_{Y}^{(n)}(t) \rightarrow S_{Y}^{0}(t)$ uniformly for $t \in[0, \infty)$. 
LEMma 4.2. $W_{2}(t)=-\int_{t}^{\infty} S^{0}(s) d S_{Y}^{0}(s)$.

Proof. Let $t$ be a continuity point of $S^{0}$. By (2.4),

$$
\begin{aligned}
W_{2}^{(n)}(t) & =-\int_{t+}^{\infty} S^{(n)}(s) d S_{Y}^{(n)}(s) \\
& =-\int_{t+}^{\infty} d\left[S^{(n)}(s) S_{Y}^{(n)}(s)\right]+\int_{t+}^{\infty} S_{Y}^{(n)}(s-) d S^{(n)}(s) \\
& =S^{(n)}(t) S_{Y}^{(n)}(t)+\int_{t+}^{\infty} S_{Y}^{(n)}(s-) d S^{(n)}(s)
\end{aligned}
$$

Hence from Lemma 4.1 we obtain

$$
\begin{aligned}
W_{2}^{(n)}(t) & \rightarrow S^{0}(t) S_{Y}^{0}(t)+\int_{t+}^{\infty} S_{Y}^{0}(s) d S^{0}(s) \\
& =-\int_{t}^{\infty} S^{0}(s) d S_{Y}^{0}(s) .
\end{aligned}
$$

Since the continuity points of $S^{0}$ are dense in $[0, \infty)$ and $W_{2}$ is continuous, we have shown $W_{2}(t)=-\int_{t}^{\infty} S^{0}(s) d S_{Y}^{0}(s), t \in[0, \infty)$.

LEMMA 4.3. $S_{Y}^{(n)}$ converges to $S_{Y}$, that is, $S_{Y}^{0} \equiv S_{Y}$.

Proof. For $t \in[0, \infty)$, let $s_{n} \in J_{n}$ such that $\left|s_{n}-t\right|=\min \left\{\left|t_{k}-t\right| ; t_{k} \in J_{n}\right\}$. Then, under Condition C, we have $s_{n} \rightarrow t$. Recall that $S_{Y}^{(n)}\left(s_{n}-\right)=\tilde{S}_{Y}^{(n)}$ $\left(s_{n}-\right)$, so

$$
\begin{aligned}
S_{Y}^{(n)}(t)-S_{Y}(t)= & {\left[S_{Y}^{(n)}(t)-S_{Y}^{(n)}\left(s_{n}-\right)\right]+\left[\tilde{S}_{Y}^{(n)}\left(s_{n}-\right)-S_{Y}\left(s_{n}-\right)\right] } \\
& +\left[S_{Y}\left(s_{n}-\right)-S_{Y}(t)\right] \\
= & I_{1 n}+I_{2 n}+I_{3 n}, \text { say. }
\end{aligned}
$$

Since $S_{Y}^{(n)} \rightarrow S_{Y}^{0}$ uniformly and $S_{Y}^{0}$ is continuous on [0, ) (Lemma 4.1), we have $I_{1 n} \rightarrow 0$. Also, $I_{2 n} \rightarrow 0$, because $\tilde{S}_{Y}^{(n)} \rightarrow S_{Y}$ uniformly on [0, $\left.\infty\right)$. Finally, $I_{3 n} \rightarrow 0$, by the continuity of $S_{Y}$. Hence (4.10) yields $S_{Y}^{(n)}(t) \rightarrow S_{Y}(t)$ for $t \in[0, \infty)$. Then $S_{Y}^{0} \equiv S_{Y}$ on $[0, \infty)$, since both $S_{Y}^{0}$ and $S_{Y}$ are continuous.

Proof of Theorem 4.1. In view of Lemmas 4.2 and 4.3 , for each $t \in[0, \infty)$ we have $W_{2}(t)=-\int_{t}^{\infty} S^{0}(s) d S_{Y}(s)$. From (2.2) this implies $\int_{t}^{\infty}\left(S^{0}(s)-\right.$ $S(s)) d S_{Y}(s)=0, t \in[0, \infty)$. Hence $S^{0}=S$ a.s. [ $\left.\lambda\right]$. But $S^{0}$ is right continuous and $S$ is continuous, so $S^{0} \equiv S$ on $[0, \infty)$. Therefore, $S^{(n)} \rightarrow S$, and the continuity of $S$ makes the convergence uniform. This completes the proof of Theorem 4.1.

Groeneboom and Wellner (1992) have shown the self-consistency of the NPMLE of $S$ and established its strong consistency without the aid of Condition C. We will prove here that, if $S^{(n)}$ is the NPMLE, then $S^{(n)}$ does satisfy Condition $\mathrm{C}$ and therefore, by Theorem 4.1, is strongly consistent. 
THEOREM 4.2. If $S^{(n)}$ is the NPMLE of $S$, then $S^{(n)}$ satisfies Condition C.

Proof. Let $F^{(n)}=1-S^{(n)}$. Then $F^{(n)}$ is the MLE of $F$. Let $Y_{(1)}<\cdots<$ $Y_{(n)}$ be the ranked $Y_{i}^{\prime}$ 's and let $\delta_{(i)}$ be the $\delta$ corresponding to $Y_{(i)}$. It is shown in Groeneboom and Wellner (1992) that the value of $F^{(n)}$ at $Y_{(i)}$ is the left derivative of $H^{*}$ at $i$, where $H^{*}$ is the convex minorant of the points $\left(i, \sum_{j \leq i} \delta_{(j)}\right)$ on $[0, n]$. Call a point $\tau \in\left\{Y_{i}: i=1,2, \ldots, n\right\}$ a vertex of the convex minorant if it satisfies $F^{(n)}(\tau)<F^{(n)}\left(Y_{i}\right)$ for any $Y_{i}>\tau$, that is, if $H^{*}$ changes its slope at $k$ if $\tau=Y_{(k)}$.

We know $F^{(n)} \rightarrow F^{0}$ at continuity points of $F^{0}$. If we could prove that $F^{0}$ is strictly increasing, then Condition $\mathrm{C}$ will be satisfied. Suppose $F^{0}$ is not strictly increasing. Then there exist $s_{1}$ and $s_{2}, s_{1}<s_{2}$, such that $F^{0}\left(s_{1}\right)=$ $F^{0}\left(s_{2}\right)$. For convenience, assume $F^{0}$ is continuous at $s_{1}$ and $s_{2}$, with $F^{0}(s)<$ $F^{0}\left(s_{1}\right)$ for any $s<s_{1}$ and $F^{0}(s)>F^{0}\left(s_{2}\right)$ for any $s>s_{2}$. Such points $s_{1}$ and $s_{2}$ can be found, because, if $F^{0}$ is not continuous at $s_{1}$ and/or $s_{2}$, we can consider $s_{1}+\varepsilon$ and/or $s_{2}-\varepsilon$ for small $\varepsilon$, and the proof is similar.

Let $s_{1 n}=\max \left\{\tau \leq s_{1}: \tau\right.$ is a vertex of $\left.F^{(n)}\right\}$ and $s_{2 n}=\min \left\{\tau \geq s_{2}: \tau\right.$ is a vertex of $F^{(n)}$ \}. Then $s_{1 n} \rightarrow s_{1}$ and $s_{2 n} \rightarrow s_{2}$, since $F^{(n)} \rightarrow F^{0}$. Therefore, since $H^{*}$ is convex minorant, we have

$$
F^{(n)}\left(s_{1}\right) \leq \frac{\text { the number of } \delta_{i} \text { 's equal to } 1 \text { in }\left(s_{1 n}, s_{2 n}\right]}{\text { the number of } \delta_{i} \text { 's in }\left(s_{1 n}, s_{2 n}\right]} \leq F^{(n)}\left(s_{2}\right) .
$$

As $n \rightarrow \infty, F^{(n)}\left(s_{1}\right) \rightarrow F^{0}\left(s_{1}\right), F^{(n)}\left(s_{2}\right) \rightarrow F^{0}\left(s_{2}\right)=F^{0}\left(s_{1}\right)$. Then in the numerator of (4.11) we get

$$
\begin{aligned}
& \frac{1}{n}\left(\text { the number of } \delta_{i} \text {, s equal to } 1 \text { in }\left(s_{1 n}, s_{2 n}\right]\right) \\
& =\frac{1}{n} \sum_{i=1}^{n}\left[T_{i} \leq Y_{i}, Y_{i} \in\left(s_{1 n}, s_{2 n}\right]\right] \\
& =-\int_{s_{1 n}}^{s_{2 n}} d W_{1}^{(n)}(y) \\
& \quad{ }_{n \rightarrow \infty} \int_{s_{1}}^{s_{2}} F(y) d F_{Y}(y),
\end{aligned}
$$

since $W_{1}^{(n)} \rightarrow W_{1}$ and $W_{1}(t)=\int_{t}^{\infty} F(s) d F_{Y}(s)$ by (2.1). Likewise the denominator of (4.11) becomes

$$
\begin{aligned}
\frac{1}{n}\left(\text { the number of } \delta_{i}^{\prime} \text { s in }\left(s_{1 n}, s_{2 n}\right]\right) & =\frac{1}{n} \sum_{i=1}^{n}\left[Y_{i} \in\left(s_{1 n}, s_{2 n}\right]\right] \\
& \rightarrow{ }_{n \rightarrow \infty} \int_{s_{1}}^{s_{2}} d F_{Y}(y) .
\end{aligned}
$$


So, as $n \rightarrow \infty$, we have

$$
\frac{\int_{s_{1}}^{s_{2}} F(y) d F_{Y}(y)}{\int_{s_{1}}^{s_{2}} d F_{Y}(y)}=F^{0}\left(s_{1}\right) .
$$

By the mean value theorem for integrals, there exists $\theta \in\left(s_{1}, s_{2}\right)$ such that $F(\theta)=F^{0}\left(s_{1}\right)$. Also, since $H^{*}$ is the convex minorant, we get

$$
\frac{\text { the number of } \delta_{i} \text { 's equal to } 1 \text { in }\left(s_{1 n}, \theta\right)}{\text { the number of } \delta_{i} \text { 's in }\left(s_{1 n}, \theta\right)} \geq F^{(n)}\left(s_{1}\right) \text {. }
$$

Following the same argument leading to (4.12), we obtain, as $n \rightarrow \infty$,

$$
\frac{\int_{s_{1}}^{\theta} F(y) d F_{Y}(y)}{\int_{s_{1}}^{\theta} d F_{Y}(y)} \geq F^{0}\left(s_{1}\right)=F(\theta) .
$$

By the mean value theorem of integrals, there exists $\theta_{1} \in\left(s_{1}, \theta\right)$ such that $F\left(\theta_{1}\right) \geq F^{0}\left(s_{1}\right)=F(\theta)$, which is contrary to the fact that $F$ is strictly increasing. Therefore, $F^{0}$ must be strictly increasing.

Acknowledgments. The authors wish to thank an Associate Editor and the referees for their critical remarks which led to many improvements in the article.

\section{REFERENCES}

ChANG, M. N. and YANG, G. L. (1987). Strong consistency of a nonparametric estimator of the survival function with doubly censored data. Ann. Statist. 15 1536-1547.

Groeneboom, P. and Wellner, J. A. (1992). Information Bounds and Nonparametric Maximum Likelihood Estimation. Birkhäuser, Basel.

Paneth, N., Pinto-Martin, J., Gardiner, J. C., Wallenstein, S., Katsikiotis, V., Hegyi, T., HiatT, M. I. and Susser, M. (1993). Incidence and timing of germinal matrix/ intraventricular hemorrhage in low birthweight infants. American Journal of Epidemiology 137 1167-1176.

PECKHAM, C. S. (1991). Children born to women with HIV-I infection: natural history and risk of transmission. The European Collaborative Study. The Lancet 337 253-260.

Pinto-Martin, J., Paneth, N., Witomski, Stern, I., Schonfeld, S., Rosenfeld, D., Rose, W., Kazam, E., Kairam, R., Katsikiotis, V. and Susser, M. (1992). The central New Jersey neonatal brain hemorrhage study: design of the study and reliability of the ultrasound diagnosis. Paediatric and Perinatal Epidemiology 6 273-284.

Rabinowitz, D., Tsiatis, A. and ARAGON, J. (1995). Regression with interval-censored data. Biometrika 82 501-513.

RÜCKER, G. and MESSERER, D. (1988). Remission duration: an example of interval censored observations. Statistics in Medicine 7 1139-1145.

Turnbull, B. W. (1976). The empirical distribution function from arbitrarily grouped, censored and truncated data. J. Roy. Statist. Soc. Ser. B 38 290-295.

WANG, Z., GARDIneR, J. C. and RAMAMOORTHI, R. V. (1994). Identifiability in interval censorship models. Statist. Probab. Lett. 21 215-222.

Biostatistics Unit

College of Human Medicine

Michigan State University

EASt LANSING, Michigan 48824 